\title{
Insight into differences in nanoindentation properties of bone
}

Naiara Rodriguez $^{\mathrm{a}^{*}}$, Michelle L. Oyen ${ }^{\mathrm{b}}$, Sandra J. Shefelbine ${ }^{\mathrm{a}}$

${ }^{\text {a }}$ Department of Bioengineering, Imperial College London, London SW7 2AZ, UK

${ }^{\mathrm{b}}$ Department of Engineering, Cambridge University, Cambridge, CB2 1PZ, UK

${ }^{*}$ Corresponding author:

Naiara Rodriguez

Department of Bioengineering, 4.28/2

Imperial College London

London SW7 2AZ, UK

Email: nr211@imperial.ac.uk

Phone: +440787 3509290 


\section{ABSTRACT}

Nanoindentation provides the ideal framework to determine mechanical properties of bone at the tissue scale without being affected by the size, shape, and porosity of the bone. However, the values of tissue level mechanical properties vary significantly between studies. Since the differences in the bone sample, hydration state, and test parameters complicate direct comparisons across the various studies, these discrepancies in values cannot be compared directly. The objective of the current study is to evaluate and compare mechanical properties of the same bones using a broad range of testing parameters. Wild type C56BL6 mice tibiae were embedded following different processes and tested in dry and rehydrated conditions. Spherical and Berkovich indenter probes were used, and data analysis was considered within the elasto-plastic (Oliver-Pharr), viscoelastic and visco-elastic-plastic frameworks. The mean values of plane strain modulus varied significantly depending on the hydration state, probe geometry and analysis method. Indentations in dry bone analysed using a visco-elastic-plastic approach gave values of $34 \mathrm{GPa}$. After rehydrating the same bones and indenting them with a spherical tip and utilizing a viscoelastic analysis, the mean modulus value was $4 \mathrm{GPa}$, nearly an order of magnitude smaller. Results suggest that the hydration state, probe geometry and the limitations and assumptions of each analysis method influence significantly the measured mechanical properties. This is the first time that such a systematic study has been carried out and it has been concluded that the discrepancies in the mechanical properties of bone measured by nanoindentation found in the literature should not be attributed only to the differences on the bones themselves, but also to the testing and analysis protocols.

KEYWORDS: Nanoindentation, Bone, Visco-elastic-plastic, Viscoelastic, Oliver-Pharr, Hydration, probe geometry 


\section{NOMENCLATURE}
$A_{C}$
contact area
$\mathrm{C}_{\mathrm{i}}$
creep function coefficients
$E_{R}$
reduced modulus
E'
plane strain modulus
$f$
viscous extent $\left(G_{\infty} / G_{0}\right)$
G
shear modulus
$\mathrm{G}_{0}$
zero-time shear modulus
$G_{\infty} \quad$ equilibrium shear modulus
$G^{\prime}$
incompressible shear modulus
h
indenter displacement
$\mathrm{h}_{\mathrm{e}}$
elastic displacement
$\mathrm{h}_{\max }$
maximum displacement
$h_{p}$
plastic displacement
$\mathrm{h}_{\mathrm{v}}$
viscous displacement
$\mathrm{H}$ hardness, resistance to plastic deformation
$\mathrm{H}_{\mathrm{C}} \quad$ contact hardness, resistance to total deformation
$\mathrm{P}$
indentation load
$P_{\max }$
peak load
S
stiffness
$\mathrm{t}$
time
$t_{c}$
creep hold time
$t_{R}$
rise time
$\alpha_{1}, \alpha_{2}, \alpha_{3}$
dimensionless geometry constant
$\eta_{\mathrm{Q}}$
indentation viscosity
V
Poisson's ratio
$\mathrm{T}_{\mathrm{i}}$
viscous-elastic time constant 
1

\section{INTRODUCTION}

2 Bone has a hierarchical structure in which the organization of its constituents at smaller

3 length scales determines the mechanical properties of the whole bone. At the tissue

4 level (sub-mm length scale) bone is composed of a matrix of mineralized collagen

5 fibrils and pores (vascular and lacunar). Unlike whole bone mechanical testing,

6 analysis of mechanical properties at the tissue scale is not affected by the size, shape,

7 and porosity of the bone, allowing for tissue level material properties to be determined.

8 Nanoindentation is a widely used technique to determine the mechanical properties of

9 bone at the tissue level (Guo and Goldstein 2000; Haque et al. 2003; Lewis and Nyman

10 2008; Oyen 2010; Rho et al. 1997; Zysset et al. 1999). In nanoindentation, a probe is

11 brought into contact with a surface, pushed into the material, and retracted, while the

12 load $(\mathrm{P})$, displacement $(\mathrm{h})$ and time $(\mathrm{t})$ are recorded. Based on these P-h-t curves,

13 multiple models exist to extract mechanical properties depending on the deformation

14 modes of the indented material. Bone is heterogeneous, anisotropic, viscoelastic and

15

poroelastic and hence, various analytical and numerical models have been developed

16

17

18

19

20

21

22

23

24

25 and adapted to determine its tissue level mechanical properties such as elastic modulus, hardness and effective (viscoelastic) viscosity (Isaksson et al. 2010; Mencik et al. 2009; Olesiak et al. 2009; Oyen 2006a). Indentations on bone with sharp probes result in plastic deformation; therefore, a viscoelastic-plastic (VEP) approach has been used for Berkovich indentations (Olesiak et al. 2009; Oyen and Cook 2003). In contrast, large spherical indenters may be used to maintain small indentation strains thus preventing yielding and plastic deformation, allowing for viscoelastic (VE) analysis (Oyen 2005, 2006a, 2007). The method that is built into most commercial indentation systems is the Oliver - Pharr (OP) method $(1992,2004)$ to extract elastic-plastic properties, neglecting any contribution from time-dependent deformation. 
All three approaches, elasto-plasic, viscoelastic, and visco-elastic-plastic, have been

used to determine bone's mechanical response, but the values of the plane strain modulus obtained from different studies vary significantly. In indentation of dry bone, where OP analysis was used, Chang et al. (2011) measured a modulus of $30.8 \pm 2.0$ GPa using a Berkovich tip, while Bushby et al. (2004) found a modulus of $18.1 \pm 2.4$ GPa with a spherical tip. The viscoelastic approach in wet bone, using spherical indentation, gave moduli as small as 2 GPa (Oyen et al. 2012). Olesiak et al. (2009) obtained values of $24.78 \pm 3.07 \mathrm{GPa}$ in dry bone, utilizing sharp indentation and using the VEP model. Since the differences in the sample preparation, hydration state, and test parameters complicate direct comparisons across the various studies, these discrepancies in values could not be compared directly.

The goal of the current study is to evaluate and compare mechanical properties of the same bones using a wide range of testing and analysis methods. The bone is indented both wet and dry, and after different embedding processes. Both spherical and Berkovich indenter probes are utilized, and data analysis is considered within the OP, VE and VEP frameworks. Thus, for the first time, direct comparisons of mechanical properties of bone measured by nanoindentation after following different testing and analysis protocols are available for analysis.

\section{MATERIALS AND METHODS}

Figure 1 shows an outline of the steps followed in the sample preparation and nanoindentation test.

\subsection{Specimen Preparation}

Tibiae from four 9 week-old female C57BL/6 mice were harvested and cleaned of surrounding soft tissue. One tibia from each mouse was cut transversally at the middiaphysis using a low speed diamond saw (Isomet, Buehler GmbH, Germany). Half of one tibia from each mouse (four halves) were fixed in $70 \%$ ethanol for 48 hours, 
dehydrated in a series of increasing concentrations of ethanol $(80,90$ and $100 \%$ for 24 , 24 and $72 \mathrm{~h}$ respectively), and changed to a xylene solution (48 h). The bones were then infiltrated in pure methyl methacrylate (MMA +a-azo-iso-butyronitrile, VRW, UK) under vacuum for 24 hours. The MMA was changed for fresh MMA and infiltrated for other 24 hours. The four half tibiae were kept in a vacuum chamber and they were let to polymerize at room temperature for two weeks.

The rest of the tibiae (one whole and one half from each mouse) were kept frozen at $20^{\circ} \mathrm{C}$ in phosphate buffered saline (PBS) gauze. Before embedding the tibiae were thawed and dried in air for an hour, embedded in low viscosity epoxy resin (EPOTHIN; Buehler, Lake Bluff, IL, USA), and allowed to cure at room temperature for 24 hours. No vacuum chamber was used to minimize the infiltration of the epoxy in the bone. The whole tibiae were also sectioned transversally at the mid-diaphysis in order to have 12 specimens (3 from each mouse) embedded in epoxy resin.

All cross-sections were polished using increasing grades of carbide papers (from P600 to P1200) and finally with diamond slurry of $3,1,0.25$ and $0.05 \mu \mathrm{m}$ particle size. The samples were cleaned ultrasonically with distilled water between each polishing step.

\subsection{Nanoindentation}

Nanoindentation studies were conducted on the tibia mid-diaphyseal cross-sections using the TI700 UBI (Hysitron, Minneapolis, MN, USA). A maximum load of $8 \mathrm{mN}$ was applied longitudinally at a constant loading rate of $0.8 \mathrm{mNs}^{-1}$ following a holding time of $30 \mathrm{~s}$ (Figure 2). Nine indents were made in each specimen for each condition with a minimum spacing of $10 \mu \mathrm{m}$ between indents.

The indentation tests were first performed on the dry PMMA-infiltrated and epoxyembedded samples using a Berkovich diamond tip. Then the epoxy-embedded specimens were rehydrated in distilled water overnight and a second set of indentations with the same load protocol was carried out with the rehydrated samples. 
78 Testing time for each sample was limited to $45 \mathrm{~min}$ to prevent sample drying. The 79 same dry-wet procedure was followed for testing with a $55 \mu \mathrm{m}$ radius spherical tip. This 80 sphere size was chosen so that the contact areas were relatively small, for comparison 81 with the Berkovich results, but sufficiently large to avoid plasticity during indentation.

\section{3. DATA ANALYSIS}

83

\subsection{Models}

84

After completing the indentation tests following the trapezoidal loading (Figure 2), P-h-t (Figure 3) plots were exported. Three different models (OP, VE and VEP) were used to fit the data and to extract mechanical properties of the material.

87

88 3.1.1. Oliver-Pharr (OP)

89 In the commonly used Oliver-Pharr approach (Oliver and Pharr 1992, 2004) the elastic modulus is calculated from the unloading curve based on the assumption that the unloading response is purely elastic. Due to the time-dependent behavior of bone, the unloading is viscoelastic; nevertheless, an attempt is made to limit the contribution of viscoelasticity by introducing 30s creep hold at peak load (Briscoe et al. 1998;

94 Chudoba and Richter 2001; Feng and Ngan 2002). unloading curve. In the current study, $80 \%$ of the unloading curve has been used to obtain the slope. The contact area $\left(A_{c}\right)$ is the projected area obtained via a calibration function. These two parameters are used to compute the reduced modulus:

$$
E_{R}=\frac{S \sqrt{\pi}}{2 \sqrt{A_{c}}}
$$


99 The reduced modulus is a combination of indenter and sample material properties.

100 However, since bone is far less stiff than the diamond tip with $(E<30 G P a)$ the reduced 101 modulus can be considered as the plane strain modulus $\left(E_{R} \sim E^{\prime}\right)$ (Olesiak et al. 2009).

102 The contact hardness or the mean supported contact stress is the peak load divided by 103 the contact area.

$$
H_{c}=P_{\max } / A_{c}
$$

\subsubsection{Viscoelastic Analysis (VE)}

Negligible plastic deformation occurs with spherical indenter tips provided that the indentation strain is smaller than the yield strain, allowing for the use of viscoelastic analysis (Oyen 2005, 2006a, 2007). In this method, a linear viscoelastic response and a non-decreasing contact area are assumed. For spherical indentations, the creep period (h-t during the holding time) is fitted by a generalized standard linear solid model

111 (Figure 3.b):

$$
h^{3 / 2}(t)=\frac{3}{8 \sqrt{R}} P_{\max }\left[C_{0}-\sum_{i}^{2} C_{i} \exp \left(-t / \tau_{i}\right) R C F_{i}\right]
$$

112 Where the radius of the sphere, $R$, and the peak load, $P_{\max }$, are test parameters; and

$113 C_{0}, C_{i}$ and $T_{i}$ are the fitting parameters. $C_{0}$ and $C_{i}$ represent the creep coefficients and

$114 \mathrm{~T}_{\mathrm{i}}\left(\eta_{i} / E_{i}\right)$ are material time constants. In this study, two Kelvin-Voigt bodies and therefore 115 two time constants $\left(I_{1}, T_{2}\right)$ have been used to represent bone creep. The dimensionless 116 ramp correction factor, $R C F_{i}$, accounts for the fact that the loading is not instantaneous

117 (rising time, $t_{R}>0$ ) and it is given by (Oyen 2007):

$$
R C F_{i}=\frac{\tau_{i}}{t_{R}}\left[\exp \left(t_{R} / \tau_{i}\right)-1\right]
$$


118 From the obtained creep coefficients, the instantaneous $G_{0}$ and long-time $G_{\infty}$ shear

119 modulus for the incompressible $(v=0.5)$ case can be computed as:

$$
\begin{aligned}
& G_{0}=\frac{1}{2\left(C_{0}-\sum C_{i}\right)} \\
& G_{\infty}=\frac{1}{2 C_{0}}
\end{aligned}
$$

120 The ratio of these two extremes $f=G_{\infty} / G_{0}$ gives an idea of the extent of the timedependent deformation, where $f=1$ signifies a perfectly elastic material and $f=0$ a 122 perfectly viscous material.

123 Since in bone $v=0.3$, the calculated incompressible $(v=0.5)$ zero-time shear modulus $124 G^{\prime}$ must be translated to $G^{u}$ via (Oyen 2005):

$G^{v}=2 G^{I}(1-v)$

125 The plane strain modulus is obtained from the incompressible instantaneous shear 126 modulus (Bembey 2006):

$$
E^{\prime}=\frac{2 G}{1-v}
$$

\subsubsection{Viscoelastic-Plastic Analysis (VEP)}

Sharp indentor tips, such as a Berkovich pyramid, result in plastic deformations and a viscoelastic-plastic analysis is appropriate (Olesiak et al. 2009). This method combines viscous, elastic and plastic quadratic elements in series (Figure 4.a) to model the full response of time-dependent materials (Oyen and Cook 2003). Using a trapezoidal loading function shown in Figure 1, the full VEP displacement-time (h-t) response is defined by equations 9-11 (Olesiak et al. 2009): the loading has a viscous-elasticplastic behaviour ( $\mathrm{h}^{\mathrm{LOAD}}$ ), the holding period is defined by a viscous response ( $\mathrm{h}^{\text {CREEP }}$ ) and the unloading is viscoelastic ( $\left.\mathrm{h}^{\mathrm{UNLOAD}}\right)$. 


$$
\begin{aligned}
& h^{L O A D}(t)=(k t)^{1 / 2}\left(\frac{2 t}{3\left(\alpha_{3} \eta_{Q}\right)^{1 / 2}}+\frac{1}{\left(\alpha_{2} E^{\prime}\right)^{1 / 2}}+\frac{1}{\left(\alpha_{1} H\right)^{1 / 2}}\right) \\
& h^{C R E E P}(t)=\frac{\left(k t_{R}\right)^{1 / 2}}{\left(\alpha_{3} \eta_{Q}\right)^{1 / 2}}\left(t-t_{R}\right)+h^{L O A D}\left(t_{R}\right) \\
& h^{U N L O A D}(t)=(k t)^{1 / 2}\left(\frac{t_{R}{ }^{3 / 2}-\left(2 t_{R}+t_{c}-t\right)^{3 / 2}}{3 / 2\left(\alpha_{3} \eta_{Q}\right)^{1 / 2}}+\frac{\left(2 t_{R}+t_{c}-t\right)^{1 / 2}-t_{R}{ }^{1 / 2}}{\left(\alpha_{2} E^{\prime}\right)^{1 / 2}}\right) \quad \mathrm{t}>\mathrm{t}_{\mathrm{R}}+\mathrm{t}_{\mathrm{C}} \\
& +h^{C R E E P}\left(t_{R}+t_{c}\right)
\end{aligned}
$$

137 The dimensionless geometric constants for a perfect Berkovich tip are $\alpha_{1}=24.5, \alpha_{2}=\alpha_{3}$

$138=4.4$ (Oyen and Cook 2003); $t_{R}, t_{c}$ stand for the rising time and holding time

139 respectively; $k$ is the loading rate $\left(k=P_{\max } / t_{R}\right)$. Fitting the displacement time $(h-t)$ curve

140 to the full VEP solution allows for the direct extraction of the indentation viscosity $\left(\eta_{Q}\right)$,

141 plane strain modulus $\left(E^{\prime}\right)$ and hardness $(H$, resistance to plastic deformation). In

142 addition, the contact hardness ( $H_{c}$, resistance to all components of deformation) can be

143 calculated for comparison purposes with the Oliver-Pharr hardness (Oyen 2006b).

$$
H_{C}=\frac{P_{\max }}{\alpha_{1}\left(h_{v}+h_{e}+h_{p}\right)^{2}}=\frac{1}{\alpha_{1}\left(\left(2 t_{R} / 3\right)\left(\alpha_{3} \eta_{Q}\right)^{-1 / 2}+\left(\alpha_{2} E^{\prime}\right)^{-1 / 2}+\left(\alpha_{1} H\right)^{-1 / 2}\right)^{2}}
$$

144

145 In the VEP model a linear creep rate is assumed for the entire hold period. However,

146 this is only an approximation, and therefore only the steady-state creep was used to

147 estimate the viscosity term:

$$
h^{C R E E P}(t)=\frac{\left(P_{\max }\right)^{1 / 2}}{\left(\alpha_{3} \eta_{Q}\right)^{1 / 2}}\left(t-t_{1}\right)+h\left(t_{1}\right)
$$

149 where $t_{1}$ is defined as $t_{1}=t_{R}+t_{d} 6$ to only consider the last $5 / 6$ of the holding period and obtain a better fit of the curve. 
151 The nonlinear least-square curve-fit function in MATLAB (Mathworks, Natick, MA) was

152 used to extract the mechanical properties from this 3-step process: i) $\eta_{Q}$ was calculated 153 by fitting the holding period; ii) knowing the indentation viscosity, E' was obtained from

154 the unloading curve; ii) finally, while these two parameters were held constant, the 155 loading curve was used to compute $H$. The viscous time constant was defined as $T_{Q}=$ $156\left(\eta_{d} E^{\prime}\right)^{1 / 2}$ and represents the characteristic time scale of the material associated with 157 the viscous-elastic-plastic response to indentation.

\section{$158 \quad$ 3.2. Deformation partitioning}

159 From the OP model, the plastic $\left(h_{\mathrm{p} \_ \text {OP }}\right)$ deformation could be approximated to the 160 displacement at zero load at the end of the test. The elastic deformation $\left(h_{\mathrm{e} \_ \text {oP }}\right)$ could

161 be defined as the difference between the maximum and final deformation.

$$
h_{p_{-} O P} \approx h\left(t_{\text {max }}\right)=h_{\text {final }}
$$

$$
h_{e_{-} O P}=h_{\max }-h_{P_{-} O P}
$$

162 In sharp indentations, the VEP model allows for the partitioning of the indentation

163 response into independent elastic $\left(h_{e_{-} V E P}\right)$, plastic $\left(h_{p_{\_} V E P}\right)$ and viscous $\left(h_{V_{-} V E P}\right)$

164 deformation components (Ferguson 2009).

$$
h_{\max }=h_{e_{-} V E P}+h_{p_{-} V E P}+h_{v_{-} V E P}
$$

165 Where each of the deformations can be defined as:

$$
\begin{aligned}
& h_{e_{-} V E P}=\sqrt{\frac{P_{\text {max }}}{\alpha_{2} E^{\prime}}} \\
& h_{p_{-} V E P}=\sqrt{\frac{P_{\text {max }}}{\alpha_{1} H}}
\end{aligned}
$$




$$
h_{v_{-} V E P}=\sqrt{\frac{P_{\max }}{\alpha_{3} \eta_{Q}}}\left(\frac{2}{3} t_{R}+t_{C}\right)
$$

166 For spherical indentations with large radius, only elastic and viscous deformations are

167 present. In the VE analysis, the displacement is defined as a function of the shear 168 modulus.

$$
h^{3 / 2}(t)=\frac{3}{16 \sqrt{R}} \frac{P_{\max }}{G}
$$

169 Hence, the displacement associated with the equilibrium modulus is the elastic

170 displacement $\left(\mathrm{h}_{\mathrm{e}_{-} \mathrm{VE}}\right)$, while the difference between this and the displacement associated with the instantaneous modulus represents the viscous deformation $\left(\mathrm{h}_{\mathrm{V} \_\mathrm{VE}}\right)$.

$$
\begin{aligned}
& h_{e_{-} V E}=\left(\frac{3}{16 \sqrt{R}} \frac{P_{\max }}{G_{\infty}}\right)^{2 / 3} \\
& h_{v_{-} V E}=h_{e_{-} V E}-\left(\frac{3}{16 \sqrt{R}} \frac{P_{\max }}{G_{0}}\right)^{2 / 3}
\end{aligned}
$$

\subsection{Statististical evaluation}

174 Mean values and standard deviations of the mechanical properties of each specimen were computed. Normality tests were carried out between these means using ShapiroWilk test. Dependent t-test was used to compare normally distributed data sets;

177 Wilcoxon signed-rank test was used for non-parametric data. A difference was considered significant when $\mathrm{p}<.05$. Statistical analysis was performed using SPSS (v. 20, SPSS Inc., Chicago, IL).

\section{RESULTS}

181 Table 1 summarizes the mean values of the mechanical properties obtained from this study. From the VEP analysis, the plane strain modulus $\left(E^{\prime}\right)$, hardness $(H)$, contact 
183

184

185

186

187

188

189

190

191

192

193

194

195

196

197

198

199

200

201

202

203

204

205

206

207

208

hardness $(H c)$, indentation viscosity $\left(\eta_{Q}\right)$ and time constant $\left(I_{1}\right)$ are measured, OP method gives the reduced modulus ( $E_{R}$, which in this case is equal to $\left.E^{\prime}\right)$ and the contact hardness $(H c)$, while from the VE approach the plane strain modulus $\left(E^{\prime}\right)$ and the extent of viscosity $(f)$ are calculated together with the time constants $\left(T_{1}, T_{2}\right)$.

The mean values of plane strain modulus, which is one parameter comparable across all models, vary significantly depending on the test method, as shown in Figure 5.

Berkovich indentations on epoxy-embedded dry bones analyzed by VEP gave a mean plane strain modulus of $33.7 \mathrm{GPa}$; while after rehydrating the same bones and indenting them with a spherical tip and utilizing a VE analysis, the mean modulus value was nearly an order of magnitude smaller, at $4.1 \mathrm{GPa}$.

\subsection{Embedding medium}

No significant differences were found between the plane strain modulus and viscosity values of dry epoxy-embedded and PMMA-infiltrated samples across the models. However, the VEP model showed that the hardness was larger for PMMA-infiltrated samples $\left(\mathrm{H}_{\mathrm{PMMA}}=2.57 \pm 0.40 \mathrm{GPa}\right)$ than for epoxy embedded ones $\left(\mathrm{H}_{\mathrm{epoxy}}=1.91 \pm\right.$ $0.56 \mathrm{GPa})$.

\subsection{Hydration state}

Plane strain modulus was significantly greater in dry specimens than in rehydrated specimens in all the cases. The VEP model showed that the hardness and the viscosity term were also significantly higher in dry specimens $\left(\mathrm{H}_{\text {dry }}=1.91 \pm 0.56 \mathrm{GPa}, \eta_{\mathrm{Q}}\right.$, dry $=$ $\left.2.53 \pm 1.62 \times 10^{15} \mathrm{~Pa} \mathrm{~s}^{2}\right)$ than in their wet counterparts $\left(\mathrm{H}_{\text {wet }}=0.47 \pm 0.11 \mathrm{GPa}, \mathrm{\eta}_{\mathrm{Q}}\right.$, wet $=$ $\left.0.50 \pm 0.28 \times 10^{15} \mathrm{~Pa} \mathrm{~s}^{2}\right)$.

\subsection{Deformation partitioning}

The elastic, plastic and viscous deformations and deformation fractions for each condition are summarized in Figure 6. Both in Berkovich and spherical indentations, the total deformation increases from dry to rehydrated conditions. However the deformation 
partitioning depends on the method used to analyze the same data. Similar

210 deformation trends are found in VEP and OP approaches: elastic deformation does not

211 vary significantly when rehydrating the samples, while plastic and viscous deformations

212 increase; unlike the viscous deformation fraction, elastic and plastic fractions vary

213 significantly from dry to rehydrated conditions. The VE model shows the highest elastic

214 and viscous deformations, showing a significant increase in both deformations from dry

215 to wet conditions. In contrast, the values of elastic and viscous fractions analyzed by

216 VE do not depend on the hydration state.

\section{5. DISCUSSION}

218 In this study, systematic investigations of the effect of a wide range of indentation

219 testing methodological options were considered for indentation of the same bone 220 samples. The results show that the measured mechanical properties depend on the 221 hydration state of the samples, the probe geometry and the model used to analyze the data. As shown in Figure 7, the plane strain modulus values obtained in this study are comparable to the wide range of values found in literature. In sharp indentations, Chang et al. (2011) measured plane strain modulus of 31 GPa for B6 mice femur embedded in epoxy. Lopez-Franco et al. (2011) found modulus of 22 GPa in NTG mice femur submerged in water. Bushby et al. (2004) had modulus of $18 \mathrm{GPa}$ for equine bone embedded in PMMA indented using a spherical tip. Spherical indentations on fully rehydrated equine bone gave modulus as small as $2 \mathrm{GPa}$ (Oyen et al. 2012) after analyzing the data using a viscoelastic approach. Until now, these discrepancies in values were considered to be mainly the result of the differences on the bones themselves. However, this study demonstrates that different methods give different results even on the same bone. 


\subsection{Wet vs dry bone}

236

237

238

239

240

241

242

243

244

245

246

247

248

249

250

251

252

253

254

255

256

257

258

259

260

261

As shown in Fig. 5, plane strain modulus was significantly higher in dry specimens than in rehydrated specimens in all the cases. This tendency is in accordance with literature (Bushby 2004; Hoffler 2005; Bembey 2006a, 2006b). The deformation partitioning (Figure 6.a) showed that in all the cases the total deformation is bigger when rehydrating the bone. It must be noted that the wet samples considered here were not immersed in fluid while testing, and therefore the differences in values of fully rehydrated samples might be larger than the ones currently measured. All the methods trend in the same direction showing the capability of nanoindentation to capture differences in hydration states.

\subsection{Probe geometry}

One of the most important experimental selections is that of probe geometry, which has been shown to influence the indentation response. Berkovich indentors have a sharp tip and the transition from elastic to plastic behavior happens almost instantaneously, indicated by the deformation partitioning which shows a plastic deformation fraction of $60-80 \%$ (Figure 6.b). In contrast, spherical tips allow extended elastic to plastic transition, which can be easily detectable by plotting P-h curves in logarithmic scale (Oyen, 2011). Figure 8 shows that in the beginning the load is proportional to the displacement instead of following the $P \sim h^{3 / 2}$ elastic law. A curve parallel to the $P \sim h$ line is associated with plastic behavior of the material. However, from the mechanics point of view, the response cannot move from a plastic regime to an elastic one. This means that the indenter tip detected the contact surface too early and this induced a first regime where the load and displacement were proportional. Hence, the measured contact displacement is overestimated and so is the contact area. This might cause an underestimation in the plane strain modulus value (Zhang et al. 2008). In the current study, the data was rejected if the initial roughness curve exceeded $5 \%$ of the maximum load. Nevertheless, roughness is the likely one cause of discrepancies 
262 between the Oliver-Pharr results for bone tested in the same condition-wet or dry263 with the two different tips.

264 Figure 6.b shows the viscous, plastic and elastic deformation fractions $-h_{v} / h_{\max }, h_{p} / h_{\max }$

265 and $h_{e} / h_{\max }-$ for both probe geometries. In Berkovich indentations the viscous

266 deformation fraction is less than $10 \%$. Hence, even if Oliver-Pharr method does not

267 capture time-dependent deformation, the deformation fractions for VEP and OP are

268 similar. On the other hand, in spherical indentations the viscous deformation is about

$26925 \%$ of the total deformation. The P-h curves in logarithmic scale (Figure 8 ) have

270 shown that there was no plasticity induced in spherical indentations but the deformation

271 partitioning in the OP case shows that the plastic deformation is dominant. This reflects

272 the limitations of the Oliver-Pharr method to measure mechanical properties of time-

273 dependent materials.

\section{5.3. Embedded versus infiltrated}

275 The embedding protocol did not result in significant differences between the plane

276 strain modulus and viscosity values across the models. This demonstrates that

277 nanoindentation measures local properties of bone. However, the VEP model showed

278 that the hardness was higher for PMMA-infiltrated samples. Unlike in epoxy samples, in

279 PMMA samples, a vacuum chamber was used to infiltrate the resin into the bone

280 pores, which could contribute to an increase in hardness.

281 5.4. Analysis method: assumptions and limitations

282 Bone is heterogeneous, anisotropic, viscoelastic, and poroelastic, with a viscoelastic

283 unloading curve (Oyen and Cook 2003). OP analysis cannot capture the viscous

284 behavior of bone. The VEP is a single time constant model and its prediction capability

285 is limited when indenting a hierarchical material with different time scales such as bone

286 (Wang and Lloyd 2010). The VE model with two time constants gives a better

287 approximation of the creep hold period than the VEP model. The time constants give 
288 information about the time scales of deformation in the material relative to the time 289 frame of the experiment.

290 All three models for data analysis here are based on the same fundamental elastic contact mechanics for indentation (Sneddon 1965). The extension of elastic to VE (Lee and Radok 1960) is the approach containing the most direct adaptation of elastic contact mechanics, and that containing the fewest simplifying assumptions. Once 294 plastic deformation is included, the picture gets more complicated. While Oliver-Pharr 295 has been shown to be accurate for stiff materials, it overestimates modulus values for polymeric materials ( $\mathrm{Ngan}$ et al. 2005; Tranchida et al. 2007), in part because of the time-dependent deformation in polymers (including bone). The VEP model used here has the most a priori assumptions. VEP assumes that the viscous, plastic and elastic deformations are in series and that the creep is linear, which is too simple to capture the more complex, multiple time constant behavior observed in bone. This results in modulus values that are the greatest when compared with either a viscoelastic or OP approach. A tendency towards modulus overestimation was observed when this model was used for characterization of polymers as well (Oyen and Cook 2003). Therefore, VEP is useful for comparison of groups within studies, but further development of this model is required before quantitative material properties can be determined.

Each of the analytical models considered here is fit to different parts of the indentation load-displacement-time response. The most direct differences observed here were for spherical indentation using Oliver-Pharr, which is a fit only to the unloading data, and VE, which is a fit only to the load-hold data. The reasons for the large discrepancy between the obtained modulus values in these two cases certainly requires further

311 detailed study in the future, but the most likely explanation is the failure of OP to 312 account for viscoelastic deformation during unloading. This study provides the most 313 direct evidence yet of the extent of this effect in materials with time-dependent 314 mechanical behavior. While many studies have advocated for a hold period at peak 
315 load to "exhaust" viscoelastic deformation and minimize the effect during unloading, the

316 results here demonstrate that this approach does not provide reliable quantitative data

317 on bone nanoindentation. Similar results were achieved by Oyen and Ko (2007) after

318 using the VEP model to generate two load-displacement curves for plane strain

319 modulus that differed by a factor of 2 and resulted in equivalent unloading stiffness

320 which would lead to a difference in modulus of only a factor of 1.2 .

321 Summarizing, the OP method could be used for a fast identification of relative

322 differences in the elastic modulus between samples. The VEP model provides an

323 estimation of the elastic, plastic and viscous contributions to the bone material behavior

324 in sharp indentations. And the VE approach can be used to analyze the creep behavior

325 of bone when there is no plasticity induced.

\section{6. CONCLUSIONS}

327 This is the first time that the same bones have been tested systematically following 328 different testing and analysis options. This study demonstrates that the tissue level 329 mechanical properties of bone measured by nanoindentation depend not only on the

330 sample itself, but also on the hydration state, probe geometry and data analysis

331 method. This is why it is complicated to compare values from different studies and care

332 must be taken when choosing the experimental and analytical options. The current

333 work shows that nanoindentation is capable of capturing trends in the mechanical

334 properties. It provides the framework to compare tissue level mechanical properties of

335 different type of bones, such as bones of different ages or pathologies.

\section{ACKNOLEDGEMENTS}

337 This study has been funded by BBSR and the Basque Government predoctoral 338 fellowship. 


\section{REFERENCES}

1. Bembey, A.K., Oyen, M.L., Bushby, A.J., Boyde, A., 2006a. Viscoelastic properties of bone as a function of hydration state determined by nanoindentation. Philosophical Magazine 86, 5691-5703.

2. Bembey, A. k., Bushby, A. j., Boyde, A., Ferguson, V. I., Oyen, M. I., 2006b. Hydration effects on the micro-mechanical properties of bone. Journal of Materials Research 21, 1962-1968.

3. Briscoe, B.J., Fiori, L., Pelillo, E., 1998. Nano-indentation of polymeric surfaces. Journal of Physics D: Applied Physics 31, 2395-2405.

4. Bushby, A. j., Ferguson, V. I., Boyde, A., 2004. Nanoindentation of Bone: Comparison of Specimens Tested in Liquid and Embedded in Polymethylmethacrylate. Journal of Materials Research 19, 249-259.

5. Chang, Y.-T., Chen, C.-M., Tu, M.-Y., Chen, H.-L., Chang, S.-Y., Tsai, T.-C., Wang, Y.-T., Hsiao, H.-L., 2011. Effects of osteoporosis and nutrition supplements on structures and nanomechanical properties of bone tissue. Journal of the Mechanical Behavior of Biomedical Materials 4, 1412-1420.

6. Chudoba, T., Richter, F., 2001. Investigation of creep behaviour under load during indentation experiments and its influence on hardness and modulus results. Surface and Coatings Technology 148, 191-198.

7. Feng, G., Ngan, A.H.W., 2002. Effects of Creep and Thermal Drift on Modulus Measurement Using Depth-sensing Indentation. Journal of Materials Research $17,660-668$.

8. Ferguson V.L., 2009. Deformation partitioning provides insight into elastic, plastic, and viscous contributions to bone material behavior. Journal of the Mechanical Behavior of Biomedical Materials 2, 364-374.

9. Guo, X.E., Goldstein, S.A., 2000. Vertebral trabecular bone microscopic tissue elastic modulus and hardness do not change in ovariectomized rats. J. Orthop. Res. 18, 333-336.

10. Haque, F., 2003. Application of Nanoindentation Development of Biomedical to Materials. Surface Engineering 19, 255-268.

11. Hoffler, C.E., Guo, X.E., Zysset, P.K., Goldstein, S.A., 2005. An Application of Nanoindentation Technique to Measure Bone Tissue Lamellae Properties. J. Biomech. Eng. 127, 1046-1053.

12. Isaksson, H., Nagao, S., Małkiewicz, M., Julkunen, P., Nowak, R., Jurvelin, J.S., 2010. Precision of nanoindentation protocols for measurement of viscoelasticity in cortical and trabecular bone. Journal of Biomechanics 43, 2410-2417.

13. Lee, E.H., Radok, J.R.M., 1960. The Contact Problem for Viscoelastic Bodies. Journal of Applied Mechanics 27, 438. 
14. Lewis, G., Nyman, J.S., 2008. The use of nanoindentation for characterizing the properties of mineralized hard tissues: State-of-the art review. Journal of Biomedical Materials Research Part B: Applied Biomaterials 87B, 286-301.

15. Lopez Franco, G.E., Blank, R.D., Akhter, M.P., 2011. Intrinsic material properties of cortical bone. J. Bone Miner. Metab. 29, 31-36.

16. Menčík, J., He, L.H., Swain, M.V., 2009. Determination of viscoelastic-plastic material parameters of biomaterials by instrumented indentation. Journal of the Mechanical Behavior of Biomedical Materials 2, 318-325.

17. Ngan, A.H.W., Wang, H.T., Tang, B., Sze, K.Y., 2005. Correcting power-law viscoelastic effects in elastic modulus measurement using depth-sensing indentation. International Journal of Solids and Structures 42, 1831-1846.

18. Olesiak, S.E., Oyen, M.L., Ferguson, V.L., 2009. Viscous-elastic-plastic behavior of bone using Berkovich nanoindentation. Mechanics of TimeDependent Materials 14, 111-124.

19. Oliver, W. c., Pharr, G. m., 1992. An improved technique for determining hardness and elastic modulus using load and displacement sensing indentation experiments. Journal of Materials Research 7, 1564-1583.

20. Oliver, W. c., Pharr, G. m., 2004. Measurement of hardness and elastic modulus by instrumented indentation: Advances in understanding and refinements to methodology. Journal of Materials Research 19, 3-20.

21. Oyen, M.L., Cook, R.F., 2003. Load-displacement Behavior During Sharp Indentation of Viscous-elastic-plastic Materials. Journal of Materials Research 18, 139-150.

22. Oyen, M.L., 2005. Spherical indentation creep following ramp loading. Journal of Materials Research 20, 2094-2100.

23. Oyen, M.L., 2006a. Analytical techniques for indentation of viscoelastic materials. Philosophical Magazine 86, 5625-5641.

24. Oyen, M.L., 2006b. Nanoindentation hardness of mineralized tissues. J Biomech 39, 2699-2702.

25. Oyen, M.L., 2007. Sensitivity of polymer nanoindentation creep measurements to experimental variables. Acta Materialia 55, 3633-3639.

26. Oyen, M.L., Ko, C.-C., 2007. Examination of local variations in viscous, elastic, and plastic indentation responses in healing bone. J Mater Sci Mater Med 18, 623-628.

27. Oyen, M.L., 2010. Handbook of Nanoindentation: With Biological Applications. Pan Stanford Publishing.

28. Oyen, M.L., 2011. Nanoindentation of Biological and Biomimetic Materials. Experimental Techniques. 
29. Oyen, M.L., Shean, T.A.V., Strange, D.G.T., Galli, M., 2012. Size Effects in Indentation of Hydrated Biological Tissues. Journal of Materials Research 27, 245-255.

30. Rho, J.Y., Tsui, T.Y., Pharr, G.M., 1997. Elastic properties of human cortical and trabecular lamellar bone measured by nanoindentation. Biomaterials 18 , 1325-1330.

31. Sneddon, I.N., 1965. The relation between load and penetration in the axisymmetric boussinesq problem for a punch of arbitrary profile. International Journal of Engineering Science 3, 47-57.

32. Tranchida, D., Piccarolo, S., Loos, J., Alexeev, A., 2007. Mechanical Characterization of Polymers on a Nanometer Scale through Nanoindentation. A Study on Pile-up and Viscoelasticity. Macromolecules 40, 1259-1267.

33. Wang, Y., Lloyd, I.K., 2010. Time-dependent nanoindentation behavior of high elastic modulus dental resin composites. Journal of Materials Research 25, 529-536.

34. Zhang, J., Niebur, G.L., Ovaert, T.C., 2008. Mechanical property determination of bone through nano- and micro-indentation testing and finite element simulation. J Biomech 41, 267-275.

35. Zysset, P.K., Edward Guo, X., Edward Hoffler, C., Moore, K.E., Goldstein, S.A., 1999. Elastic modulus and hardness of cortical and trabecular bone lamellae measured by nanoindentation in the human femur. Journal of Biomechanics 32 , 1005-1012. 


\section{FIGURE CAPTIONS}

Figure 1:

Outline of the methods. Tibiae of four B6 mice were harvested and cut in half. One of the halves was dehydrated in ethanol and infiltrated with PMMA using vacuum. The other three halves were dried in air and embedded in epoxy resin. The PMMA samples were tested only in dry conditions and the epoxy ones in dry and wet conditions. For each condition, nine indents were made both with a Berkovich indentor and a sphere.

Figure 2:

Trapezoidal load function. Loading to the peak load $\left(P_{\max }=8 \mathrm{mN}\right)$ during rise time $\left(t_{R}=\right.$ $10 \mathrm{~s})$ with a creep hold $\left(t_{c}=30 \mathrm{~s}\right)$ before unloading. Same loading and unloading rate $\left(k=P_{\max } / t_{R}\right)$.

Figure 3:

Load-displacement (a) and displacement-time (b) plots obtained after applying the trapezoidal loading protocol in Figure 2 on a rehydrated sample using a Berkovich indenter probe.

Figure 4:

Rheological model for (a) VEP on loading (adapted from Oyen and Cook 2003); and (b) VE during creep hold.

Figure 5:

Mean plane strain modulus and standard deviations for Berkovich indentations analyzed by VEP and OP and spherical indentations modeled with OP and VE in dry and rehydrated conditions.

Figure 6:

Viscous $h_{v}$, plastic $h_{p}$, and elastic $h_{e}$ mean deformations (a) and mean deformation fractions (b) of Berkovich and spherical indentations in dry and rehydrated conditions.

Figure 7:

Comparison of the mean plane strain modulus of the current study (outlined) with other studies on dry and wet bone indented using Berkovich and spherical indenter probes. The analysis method used in each study is specified (OP, VE or VEP). The values obtained in this study for the same bones are comparable with the wide range found in the literature for different animal bones.

Figure 8:

Logarithmic curve of P-h data for a spherical indent on dry bone embedded in epoxy together with $\mathrm{P} \sim \mathrm{h}$ (plastic behavior) and $\mathrm{P} \sim \mathrm{h}^{3 / 2}$ (elastic behavior) curves. 


\begin{tabular}{|c|c|c|c|c|c|}
\hline & \multicolumn{2}{|c|}{ Berkovich } & \multicolumn{2}{|c|}{ Sphere $(55 \mu \mathrm{m})$} \\
\hline & & VEP & OP & VE & OP \\
\hline \multirow{6}{*}{$\begin{array}{l}\text { Dry } \\
\text { pmma }\end{array}$} & $E^{\prime}[\mathrm{GPa}]$ & $36.4 \pm 9.0$ & $22.9 \pm 3.7$ & $7.2 \pm 2.6$ & $15.4 \pm 3.7$ \\
\hline & $\mathrm{H}[\mathrm{GPa}]$ & $2.57 \pm 0.40$ & & & \\
\hline & $\mathrm{Hc}$ [GPa] & $0.93 \pm 0.06$ & $0.93 \pm 0.07$ & & $0.17 \pm 0.05$ \\
\hline & 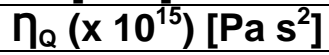 & $2.96 \pm 1.86$ & & & \\
\hline & $\mathbf{f}=\mathbf{G}_{\infty} / \mathbf{G}_{0}$ & & & $0.63 \pm 0.04$ & \\
\hline & $\mathbf{T}_{1}, \mathbf{T}_{2}[\mathbf{S}]$ & $277.2 \pm 64.7$ & & $\begin{array}{l}2.0 \pm 0.8 \\
19.6 \pm 14.6\end{array}$ & \\
\hline \multirow{6}{*}{$\begin{array}{l}\text { Dry } \\
\text { epoxy }\end{array}$} & $E^{\prime}[\mathrm{GPa}]$ & $33.7 \pm 6.4$ & $20.1 \pm 3.9$ & $6.6 \pm 2.0$ & $11.6 \pm 1.7$ \\
\hline & $\mathrm{H}[\mathrm{GPa}]$ & $1.91 \pm 0.56$ & & & \\
\hline & $\mathrm{Hc}[\mathrm{GPa}]$ & $0.75 \pm 0.16$ & $0.74 \pm 0.19$ & & $0.15 \pm 0.05$ \\
\hline & $\eta_{\mathrm{Q}}\left(\mathrm{x}_{10^{15}}\right)\left[\mathrm{Pa} \mathrm{s} \mathrm{s}^{2}\right]$ & $2.53 \pm 1.62$ & & & \\
\hline & $\mathbf{f}=\mathbf{G}_{\infty} / \mathbf{G}_{0}$ & & & $0.54 \pm 0.13$ & \\
\hline & $\mathbf{T}_{1}, \mathbf{T}_{2}[\mathbf{s}]$ & $252.0 \pm 73.4$ & & $\begin{array}{l}2.0 \pm 0.7 \\
18.8 \pm 11.5\end{array}$ & \\
\hline \multirow{6}{*}{$\begin{array}{l}\text { Wet } \\
\text { epoxy }\end{array}$} & $E^{\prime}$ [GPa] & $27.5 \pm 6.5$ & $11.5 \pm 2.0$ & $4.1 \pm 1.4$ & $9.2 \pm 2.4$ \\
\hline & $\mathrm{H}[\mathrm{GPa}]$ & $0.47 \pm 0.11$ & & & \\
\hline & $\mathrm{Hc}$ [GPa] & $0.26 \pm 0.04$ & $0.23 \pm 0.03$ & & $0.10 \pm 0.04$ \\
\hline & $\eta_{\mathrm{Q}}\left(\mathrm{x}_{10^{15}}\right)\left[\mathrm{Pa} \mathrm{s} \mathrm{s}^{2}\right]$ & $0.50 \pm 0.28$ & & & \\
\hline & $\mathbf{f}=\mathbf{G}_{\infty} / \mathbf{G}_{0}$ & & & $0.51 \pm 0.08$ & \\
\hline & $\mathbf{T}_{1}, \mathbf{T}_{2}[\mathbf{s}]$ & $133.3 \pm 39.0$ & & $\begin{array}{l}2.0 \pm 0.6 \\
17.3 \pm 9.0\end{array}$ & \\
\hline
\end{tabular}

Table 1: Summary of means and standard deviations of tissue mechanical properties according to the probe geometry and data analysis method. E' is plane strain modulus; $\mathrm{H}$ is hardness (resistance to plastic deformation; $\mathrm{Hc}$ is contact hardness (resistance to deformation); $\eta_{\mathrm{Q}}$ is indentation viscosity; $f$ represents the elastic fraction (viscous, $0 \leq f$ $\leq 1$, elastic); and $\mathrm{T}_{1}, \mathrm{~T}_{2}$ are viscoelastic time constants (one time constant for VEP and two for VE). 
348 FIGURES

349 Figure 1:

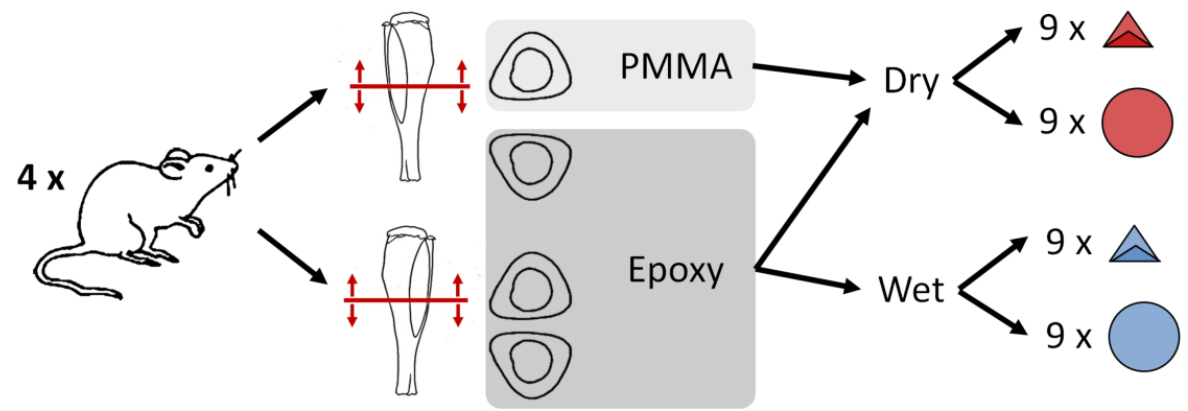

Figure 2:

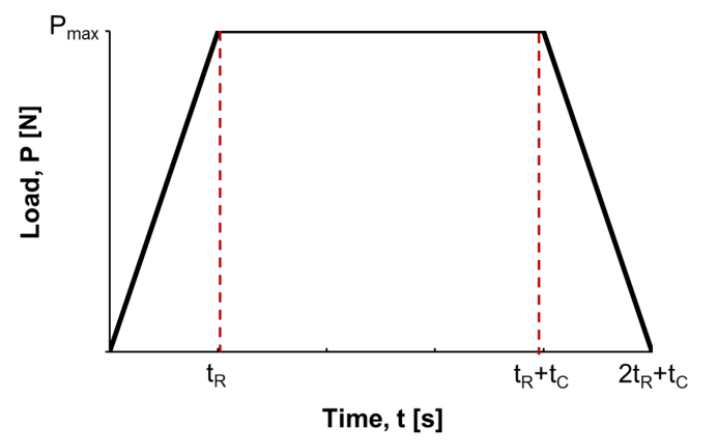

Figure 3:

a)

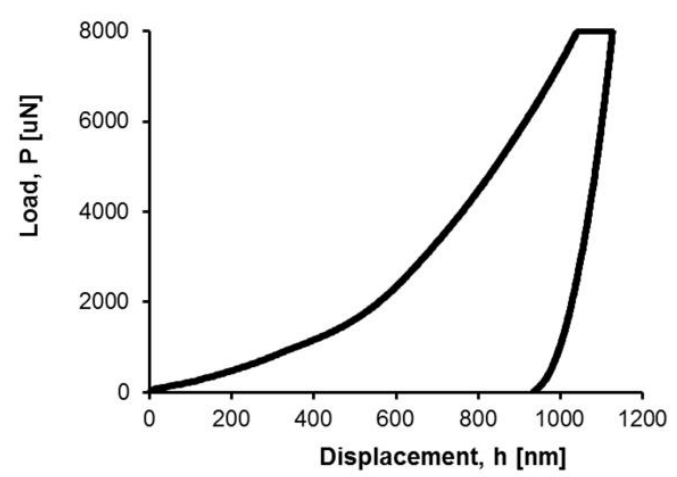

b)

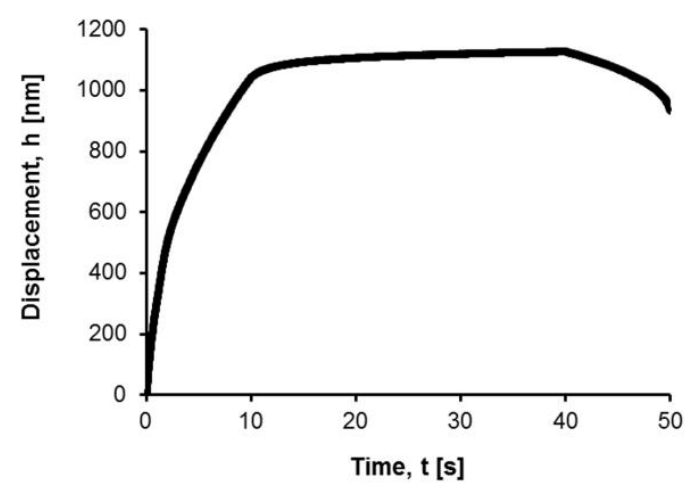


Figure 4:

a)

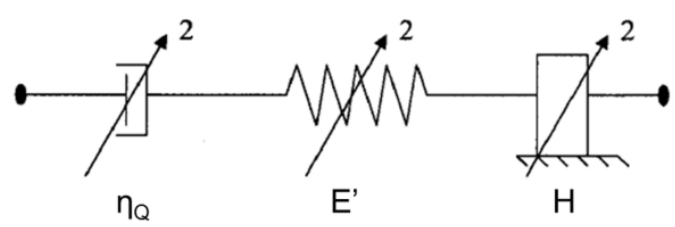

b)

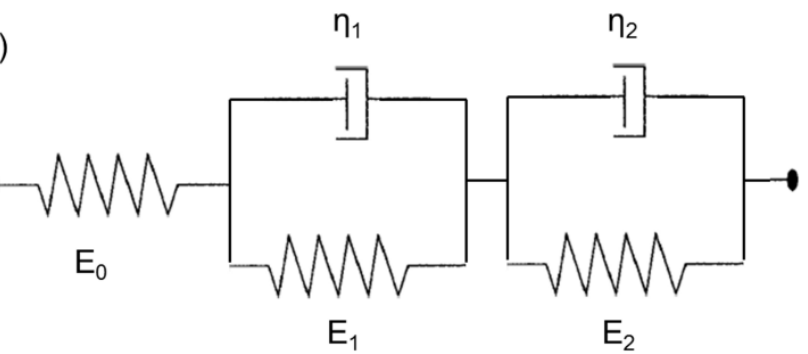

Figure 5:

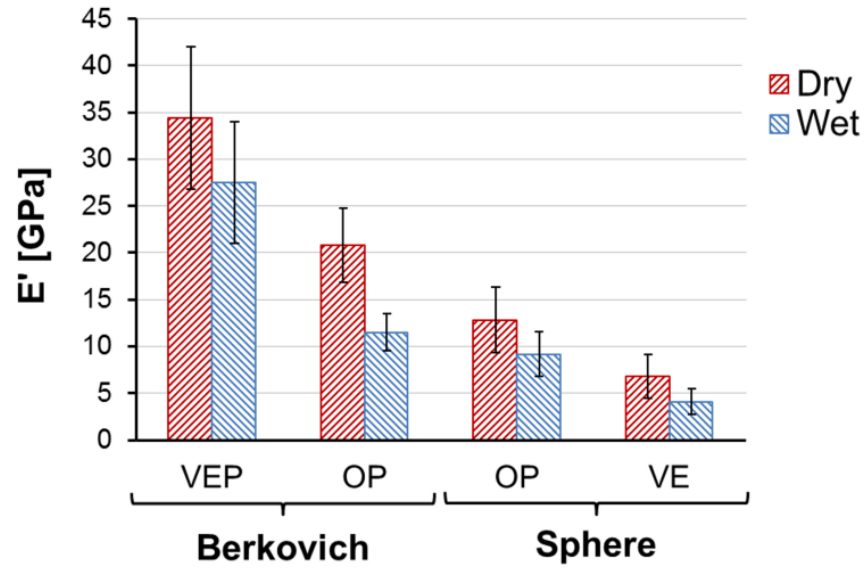


Figure 6:

a)

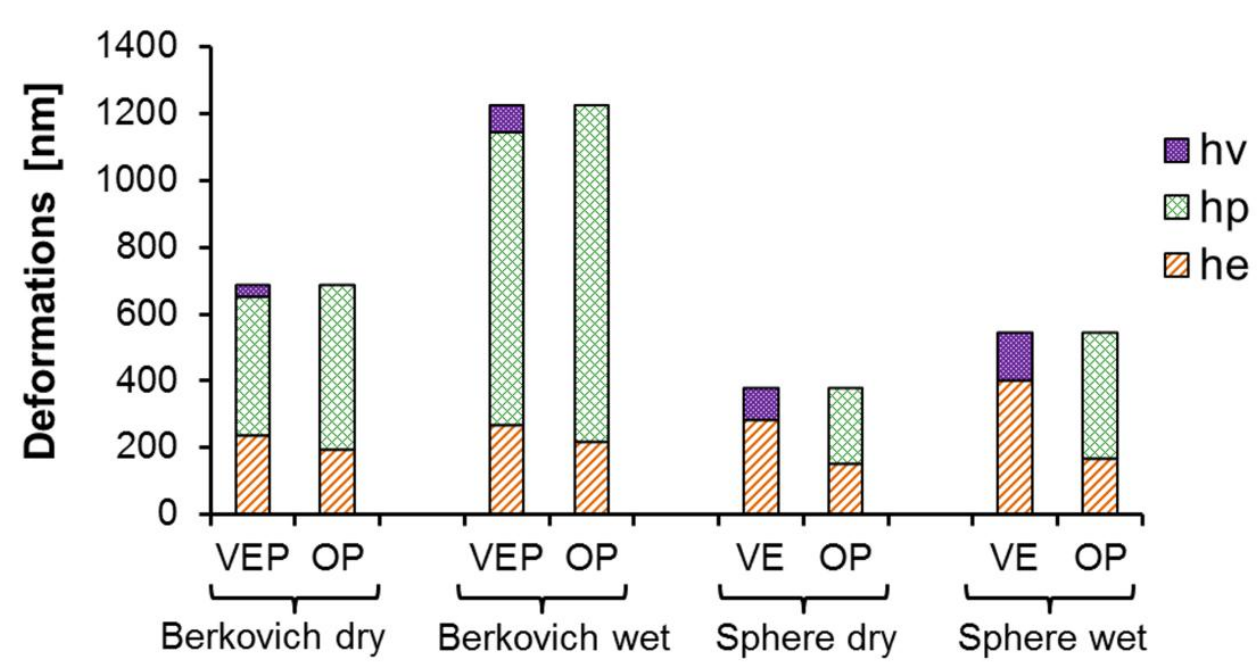

b)

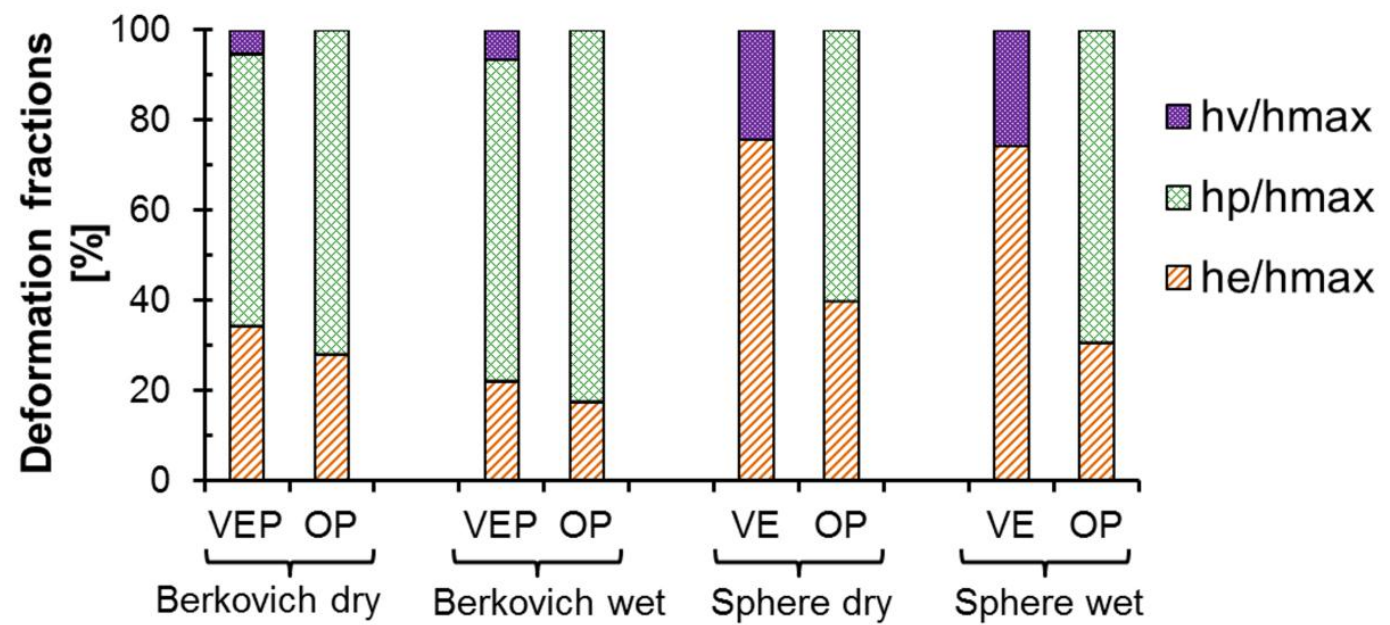


Figure 7:
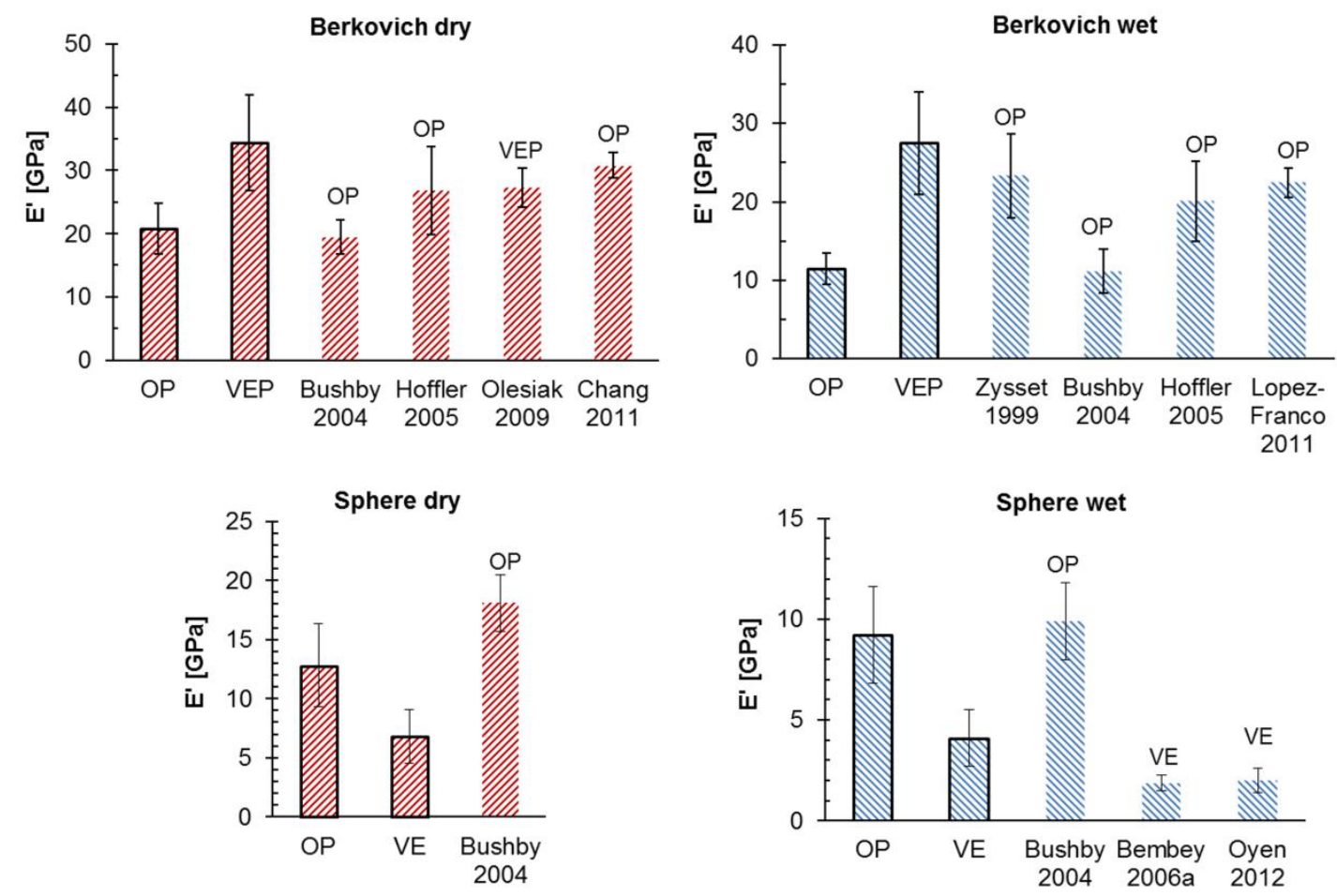

Figure 8:

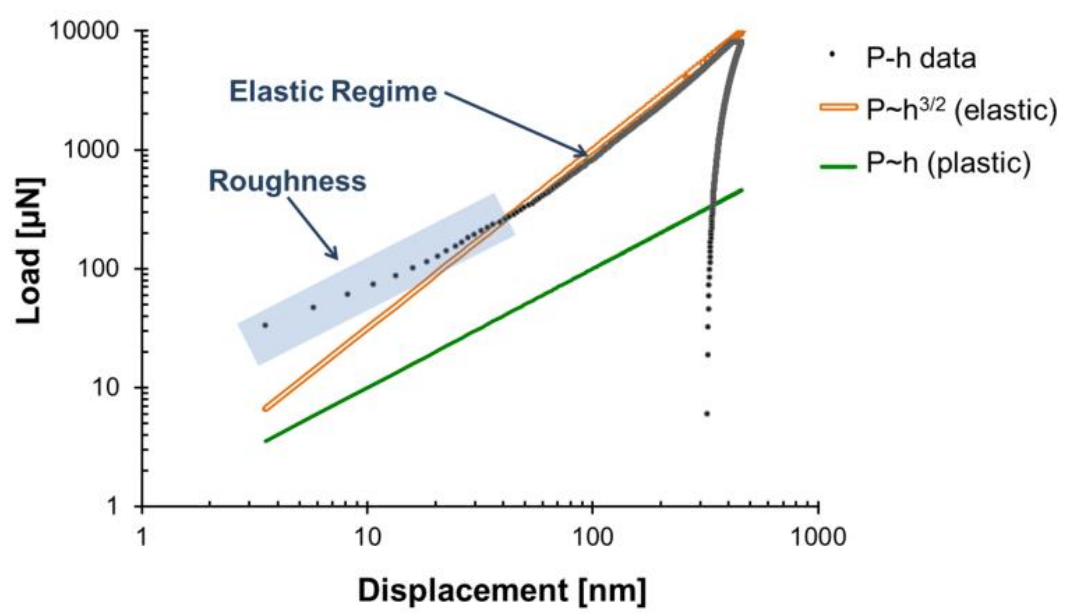

